\title{
Intravesical treatment with highly-concentrated hyaluronic acid and chondroitin sulphate in patients with recurrent urinary tract infections: Results from a multicentre survey
}

\author{
Antonio Cicione, MD; ${ }^{*}$ Francesco Cantiello, MD; ${ }^{*}$ Giuseppe Ucciero, MD; ${ }^{*}$ Andrea Salonia, MD; ${ }^{\dagger}$ \\ Marco Torella, MD; $;$ Marco De Sio, MD, $;$ Riccardo Autorino, MD, $;$ Antonio Carbone, MD,f \\ Martin Romancik, MD,; Roman Tomaskin, MD,: Rocco Damiano, MD*
}

*Department of Urology \& Research Doctorate Urology Program, Magna Graecia University, Catanzaro, Italy; 'Department of Urology, University Vita-Salute San Raffaele, Milano, Italy; ${ }^{\S}$ Gynaecology Unit, Second University of Naples, Napoli, Italy; *Urology Unit, Second University of Naples, Napoli, Italy; £Unit of Urology - ICOT, Sapienza University of Rome, Latina, Italy; " Department of Urology, St. Cyril and Method University Hospital, Bratislava, Slovakia; ${ }^{ \pm D}$ epartment of Urology, Faculty Hospital of Martin (MFN), Martin, Slovakia

Cite as: Can Urol Assoc J 2014;8(9-10):e721-7. http://dx.doi.org/10.5489/cuaj.1989

Published online October 22, 2014.

\section{Abstract}

Introduction: We assess the effectiveness of intravesical instillation of hyaluronic acid (HA) and chondroitin sulphate (CS) as a non-antibiotic treatment option for prophylaxis of recurrent urinary tract infections (UTIs) in female patients.

Methods: This was a retrospective cohort study involving 7 European institutions. We included patients with recurrent UTIs who received intravesical instillations of laluril (IBSA International) (50 mL HA 1.6\% and CS 2\% solution) between January 2010 and March 2012. Medication schedule, length of follow-up, recurrence infection time, number of UTIs/patients/year, patient quality of life, subjective symptoms score, and treatment-emergent side effects were recorded and analyzed.

Results: In total, 157 women (mean age: $54.2 \pm 4.1$ years) were included in the analysis. All patients had at least 12 months followup. After 4 weekly and 5 monthly HA-CS bladder instillations, UTI episodes decreased from $4.13 \pm 1.14$ to $0.44 \pm 0.50(p=0.01)$ at 12 months, while recurrent UTI time prolonged from $94.8 \pm 25.1$ days to $178.4 \pm 37.3$ days $(p=0.01)$ at 12 months. An improvement in symptoms and quality of life was achieved. A medium-depth pain after medication instillation was the most reported side effect. Regression model analysis showed significant risk factors in developing new UTI episodes: being more than 50 years old and having more than 4 UTI episodes per year (OR 3.41; CI 95\%; 1.51-7.71, $p=0.003$ and OR 3.31; $\mathrm{Cl} 95 \% 1.51-7.22 ; p=0.003$, respectively). Retrospective design and lack of a control group represent two main limitations of the study.

Conclusions: Restoring glycosaminoglycans bladder layer therapy is a promising non-antibiotic therapy to prevent recurrent UTIs.

\section{Introduction}

According to the European Association of Urology (EAU) guidelines, recurrent urinary tract infections (UTIs) are defined as the presence of lower urinary tract symptoms and at least 3 episodes of documented positive urine culture $>10^{3}$ colonies $/ \mathrm{mL}$ on a voided urine volume specimen in the previous 12 months. ${ }^{1}$

Uncomplicated recurrent UTIs are extremely common. ${ }^{2}$ Among healthy young women, urinary infections recur in $25 \%$ of cases within 6 months after the first UTI. ${ }^{3}$ Recurrent UTIs are experienced by as many as $5 \%$ of women at some time during their life, with an increasing recurrence rate in women with more than 1 prior UTI. ${ }^{4}$

Antibiotics are routinely prescribed for UTI, with the primary goal being a rapid resolution of urinary symptoms. An antimicrobial prophylaxis given for 6 consecutive months is suggested by the EAU Guidelines as a therapeutic option for women with recurrent UTIs when behavioural modifications and non-antimicrobial measures, such as cranberry juice or probiotics, are unsuccessful. ${ }^{1}$

According to a Cochrane systematic review, ${ }^{5}$ continuous low-dose antimicrobial prophylaxis is effective in preventing UTIs, with a 0.15 risk ratio (RR) for clinical recurrence per patient per year. However, continuous low-dose antimicrobial prophylaxis also presents a $1.58 \mathrm{RR}$ for severe treatmentemergent side effects, such as vaginal/oral candidiasis and gastrointestinal symptoms; these symptoms eventually lead to treatment withdrawal. Furthermore, patients with history of recurrent UTIs have a higher incidence of sexual pain, secondary provoked vestibulodynia, ${ }^{6}$ and irritative urinary symptoms even when they are infection-free. ${ }^{7}$

Research in the field of glycosaminoglycans (GAGs) started in $1970,{ }^{8}$ but only recently has there been an increasing 
number of preclinical and clinical studies advocating a significant role of GAGs disorders in the pathogenesis of many chronic bladder diseases, such as bacterial recurrent UTIs, interstitial cystitis, overactive bladder, and bladder cancer. ${ }^{9}$ Parsons and colleagues, ${ }^{10}$ in an animal study, identified a GAGs layer covering the superficial layer of urothelium as responsible for the antibacterial defense mechanism of the bladder. A domino-like theory was postulated as cause of the chronic course of bladder disease, where GAGs layer loss that fails to heal was the first step leading to direct exposure of epithelial cells to urine components, facilitating bacterial adherence and infection ${ }^{9,11}$ and chronic bladder inflammation. ${ }^{12}$

Considering the loss of urothelium GAGs layer as a key factor promoting recurrent UTIs, a therapy aimed at restoring GAGs layer, based on the combination of hyaluronic acid (HA) and chondroitin sulphate (CS), was conceived and recently suggested as an option to prevent recurrent cystitis.

To date, few clinical studies support the benefit of $\mathrm{HA}$ and CS in this setting. ${ }^{13-17}$ Although a positive effect was reported, a small sample was considered the main limitation of the studies supporting the use of GAGs to prevent UTI. More robust analyses of larger samples are required to corroborate positive findings from early series. ${ }^{14-17}$

This study was initiated as a collaborative effort to provide a review of clinical outcomes from centres pioneering the intravesical instillation of laluril (IBSA International) (HA $1.6 \%$ plus CS $2 \%$ ) for prophylaxis of recurrent urinary tract infections (UTIs) in female patients.

\section{Methods}

\section{Study design}

This was a retrospective cohort multicenter study involving 7 European centers. The study was approved by the Magna Graecia University institutional review board and a datasharing agreement was obtained from each participating site.

Clinical data and outcomes of patients who had undergone to laluril instillations between January 2010 and March 2012 were collected and gathered into a dedicated datasheet.

\section{Inclusion and exclusion criteria}

Eligible patients were women with a documented history of bacterial recurrent UTIs, defined as at least 3 episodes of uncomplicated UTIs with the isolation of $>10^{3} \mathrm{CFU} / \mathrm{mL}$ of an identified pathogen with clinical symptoms in the last 12 months. $^{1}$

Patients were excluded if they had $<3$ uncomplicated UTIs in the previous year, significant $(>50 \mathrm{~mL}$ ) post-voidal residual urine, neurologic bladder disease, known bladder neoplasia, urinary stone or abnormality of the urinary tract, renal insufficiency, diabetes mellitus, current corticosteroid use, current immunosuppressive disease, use of spermicides or intrauterine devices, current pregnancy or if they were on an antibiotic prophylaxis regimen.

\section{Treatment protocol}

Treatment protocol consisted of intravesical instillations of $50 \mathrm{~mL}$ laluril (HA $1.6 \%$ and CS $2 \%$ ), weekly for 4 weeks (induction phase), and then monthly for 5 months (maintenance phase). Three days before each intravesical instillation, urinalysis and urine culture were performed. In case of asymptomatic bacteruria or UTI, the treatment was delayed and an antibiotic treatment was administered just in UTI cases.

\section{Assessment}

The following variables were collected: demographic data, including age, body mass index, menopausal status, and history of constipation (defined as having a bowel movement fewer than three times per week); disease-related features, including UTI frequency, UTI recurrence time over the last year, previous pathogens involved in recurrent UTIs; history of prescribed antibiotics; cystoscopic findings; and uroflowmetry findings. Cystoscopy was performed without antibiotic prophylaxis.

After enrollment, patients were monitored at 1 month (V1) and then every 6 months up to 24 months (V2, V3, V4, V5). At every visit patients completed the Pelvic Pain and Urinary/Frequency (PUF) patient symptom scale ${ }^{18}$ and the quality of life (QoL) Short Form (SF)-36 questionnaires ${ }^{19}$ to assess symptom score and quality of life, respectively. Moreover, urinalysis and urine culture were performed before each bladder instillation and at each follow-up visit, both in healthy and symptomatic patients, through a cleancatch midstream urine sample, to appraise UTI episodes, as defined by EAU guidelines. ${ }^{1}$ We also recorded other therapies given to patients during treatment or during the follow-up period.

The following treatment outcomes were analyzed: treatment-related side effects; pain/discomfort at weekly and monthly instillations end (V1 and V2); treatment efficacy assessed by calculating any reduction of UTI episodes per patient per year, length of time to UTI recurrence (defined as the time elapsed between the first instillation and the first recurring UTI), and by impact of therapy on QoL and urinary symptoms. 


\section{Statistical analysis}

Normal distribution of continuous variables was initially tested by Kurtosi test. Mean and standard deviation (SD) were used for descriptive statistics. The statistical significance of differences in means and proportions was tested with the 2-tailed $t$ test and the Pearson $\chi^{2}$ test, respectively. Kaplan Meier method tested time to UTI recurrence, considering a 12-month time frame before and after treatment. Binary logistic regression analyses tested the effect of various independent variables on UTI development. All statistical analyses were performed using SPSS 18.0 for windows (SPSS Inc., IBM Corp., Somers, NY). All tests were two-sided, with a significance level set at 0.05.

\section{Results}

\section{Study sample and intervention}

Overall, 157 patients were included in the study (Table 1). A course of 4 instillations over 4 consecutive weeks (induction phase) followed by 5 monthly instillations (maintenance phase) was the main HA-CS treatment schedule, although $36(28 \%)$ patients received a different regimen due to further instillations up to 12 months. Delaying instillations for positive urine culture was needed in 23 cases $(14.6 \%)$.

\section{Pre-treatment assessment}

Mean total PUF and symptom score were 20.09 (5.7) and 13.7 (3.78), respectively, whereas the mean QoL SF-36 score was 59.25 (11.1). At cystoscopy, a bladder hyperemia was reported in 74 patients $(47.1 \%)$.

\section{Follow-up}

A 12-month follow-up was available for all patients, with 41 patients $(26.1 \%)$ having a longer (24 months) follow-up.

\section{Outcome assessment}

At 12 months, few patients have had new UTIs, but an increasing trend in UTIs was recorded at 24 months, with 41 patients reporting up to 139 UTIs episodes. E. Coli represented the most frequent uropathogen, mainly requiring a 3-day course of quinolones.

The estimating time-to-UTI recurrence at the 12-month follow-up was longer compared with the pre-treatment value: 178.4 (37.3) versus $94.8(25.1)$ days $(p=0.01)$ (Fig. 1). Similarly, the computed number of UTIs was lower than before treatment: $0.44(0.50)$ versus 4.13 (1.14) episodes/ person/year $(p=0.01)$.
Table 1. Individual patient characteristics

$\begin{array}{lcc} & \text { Mean (SD) } & \text { No. (\%) } \\ \text { Patients, no. } & 157 & \\ \text { Age, yr } & 53.1(13.4) & \\ \text { BMI, kg/m2 } & 25.1(3.4) & \\ \text { Affected with constipation } & 73(46.4) \\ \text { Menopausal stage } & & 71(45.2) \\ \text { Follow-up length } 12 \text { months } & 157 & \\ \text { UTI episodes over last year, } & 4.13(1.14) & \\ \begin{array}{l}\text { UTI recurrence time over last year, } \\ \text { days }\end{array} & 94.8(25.1) & \end{array}$
days

UTI agent, E. coli/other

$(84.7) / 24$

(15.3)

Advised medication, quinolone/ other

(B) 3-day void urine diary

(13.4)

Omax, mL/sec

$16.3(4.6)$

Qave, $\mathrm{mL} / \mathrm{sec}$

$24.05(4.6)$

PVR, $\mathrm{mL}$

$12.0(3.2)$

$18.8(16.4)$

Bladder mucosa hyperemia at cystoscopy 1

$74(47.1)$

Total PUF score

$20.09(5.7)$

Symptom score

13.7 (3.78)

QoL SF-36 score

59.25

(11.1)

Instillations schedule, 4 weekly +

5 monthly/different

$121(77) / 36$

(23)

One or more additional

(C) instillations

$36(23)$

Regimen weekly/biweekly

$29 / 7$

Delayed instillations due to urine

culture positivity status, no. cases

$23(14.6)$

BMI; body mass index; UTI: urinary tract infection; PVR: post-void residual volume; PUF: pelvic pain and urinary/frequency patient symptom scale; QoL: quality of life; SD: standard deviation. Data of continuous variables are expressed as means (standard deviation) while for categorical variables as count and (\% percentages). 1Defined as: bladder mucosa area reddened despite the close mucosa.

A significant improvement in the PUF and the SF-36 scores during the first 12 months (Table 2, Fig. 2) was noted, with a subsequent worsening of symptoms or QoL, respectively, over the subsequent 12 months.

Overall, 10 patients reported moderate storage urinary symptoms in the absence of infection, but only 1 required medication for symptom relief. Patients reported moderate pain for 2 days after both weekly (mean visual analogue scale [VAS] 4.5) and monthly (mean VAS 4.2) instillations.

At logistic regression analysis, the following factors were predictors of new UTI episodes: age over 50 (odds ratio [OR] $3.41 ; 95 \%$ confidence interval $[\mathrm{Cl}] 1.51-7.71 ; p=0.003$ ) and having more than $4 \mathrm{UTI}$ episodes per year (OR 3.31; $95 \% \mathrm{Cl}, 1.51-7.22 ; p=0.003$ ) (Table 3 ). 
Cicione et al.

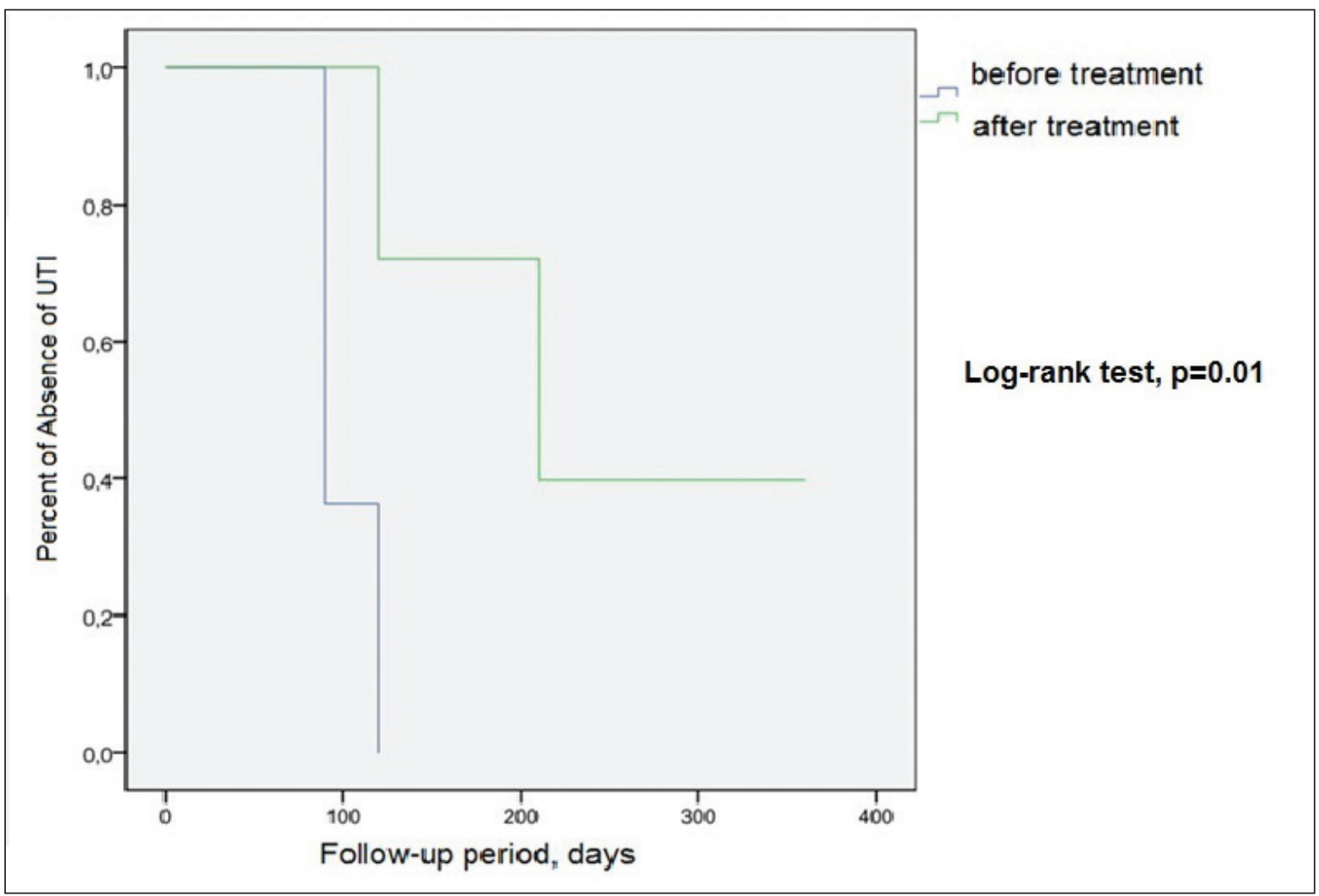

Fig. 1. Kaplan-Meier plotting of time to recurrence of infection.

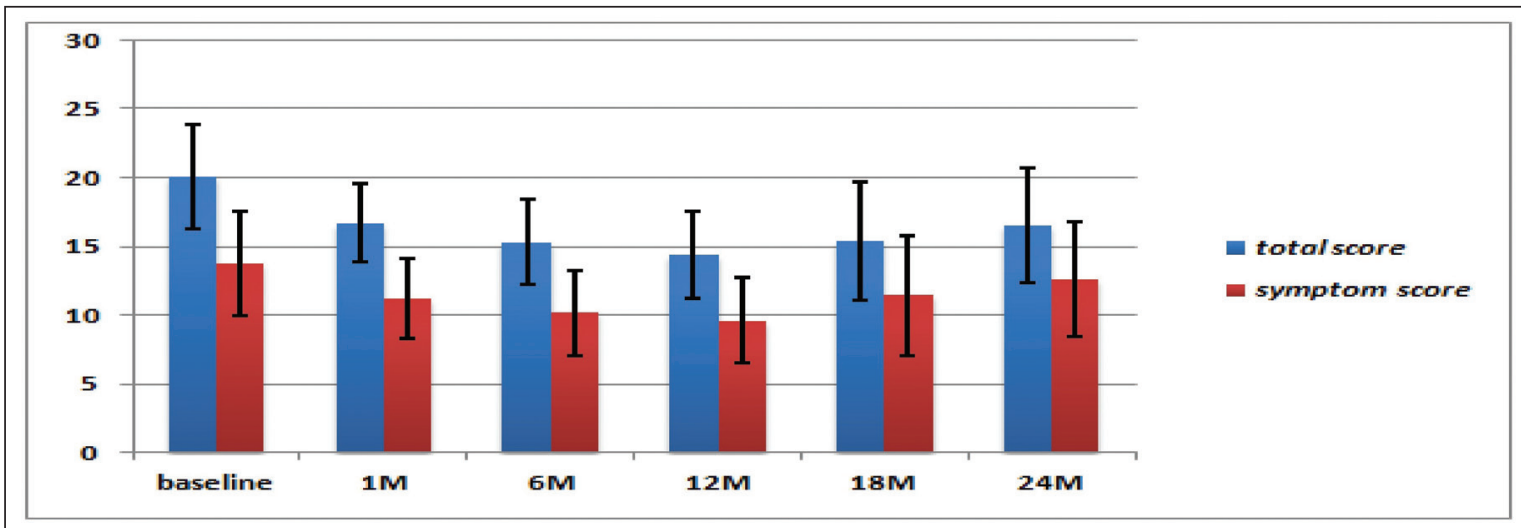

SF 36 score

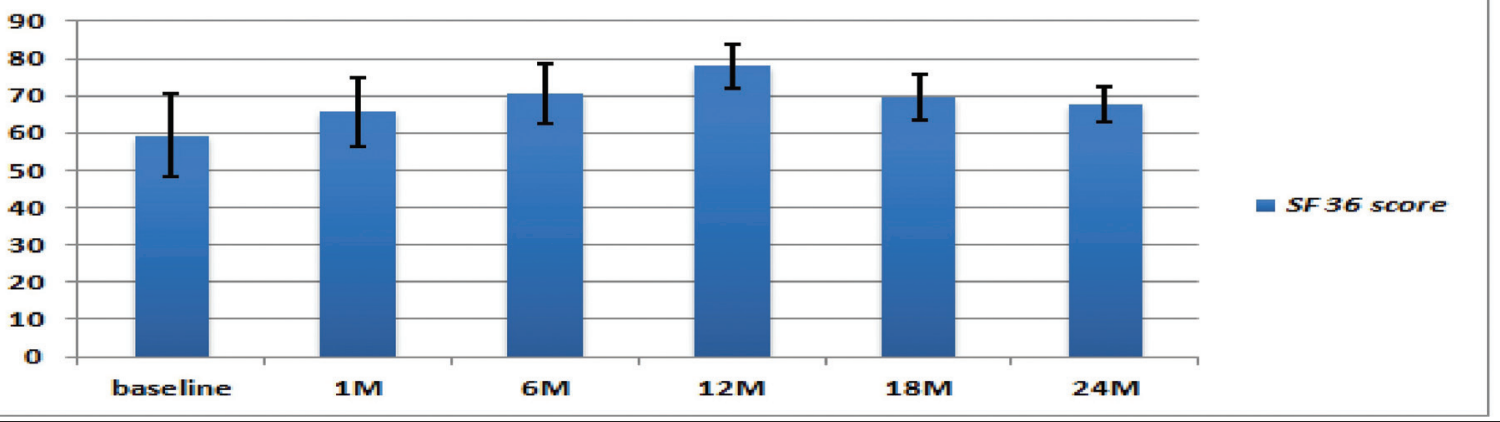

Fig. 2. Pelvic pain and urinary/frequency symptoms, total score and SF-36 quality of life score during patient follow-up. 
Intravesical GAGs treatment in patients with recurrent UTIs

\begin{tabular}{|c|c|c|c|c|c|c|}
\hline Follow-up clinic visit & V1 at 1 month & V2 at 6 months & V3 at 12 months & V4 at 18 months & V5 at 24 months & $p$ value* \\
\hline No. evaluated patients & 157 & 157 & 157 & 71 & 41 & $\mathrm{n} / \mathrm{a}$ \\
\hline Reported UTla episodes & 2 & 36 & 69 & 130 & 139 & $\mathrm{n} / \mathrm{a}$ \\
\hline Patients with UTla, no. (\%) & $2(1 \%)$ & $30(22.3 \%)$ & $22(18.1 \%)$ & $22(30.9 \%)$ & $15(36.5 \%)$ & 0.001 \\
\hline $\begin{array}{l}\text { Mean rate of UTla episodes/ } \\
\text { patient }\end{array}$ & $0.01(0.11)$ & $0.23(0.42)$ & $0.44(0.50)$ & $1.83(1.02)$ & 3.39 (1.79) & 0.01 \\
\hline $\begin{array}{l}\text { Pathogen involved: E. Coli/ } \\
\text { others }\end{array}$ & $2 / 0$ & $21 / 9$ & $19 / 3$ & $18 / 4$ & $13 / 2$ & 0.001 \\
\hline $\begin{array}{l}\text { Antibiotic regimen prescribed } \\
\text { (quinolones/others) }\end{array}$ & $2 / 0$ & $24 / 6$ & $18 / 4$ & $14 / 4$ & $14 / 1$ & 0.001 \\
\hline Total PUF score & $16.7(3.87)$ & $15.3(4.95)$ & $14.4(4.61)$ & $15.4(3.61)$ & $16.5(3.91)$ & 0.01 \\
\hline Symptom score & $11.2(2.89)$ & $10.173 .08)$ & $9.6(3.13)$ & $11.4(4.32)$ & $12.6(4.18)$ & 0.01 \\
\hline SF-36 score & $65.6(9.1)$ & $70.39(8.01)$ & $77.92(5.89)$ & $69.52(6.19)$ & $67.5(4.71)$ & 0.01 \\
\hline Adverse events & & & $\mathrm{n} / \mathrm{a}$ & $\mathrm{n} / \mathrm{a}$ & $\mathrm{n} / \mathrm{a}$ & \\
\hline Patients, no. (\%) & $2(1 \%)$ & $8(5 \%)$ & & & & \\
\hline \multicolumn{7}{|l|}{ Mean VAS score } \\
\hline \multicolumn{7}{|l|}{ Minimal (VAS 1-3) } \\
\hline Moderate (VAS 4-7) & $4.5(0.7)$ & $4.2(0.5)$ & & & & 0.87 \\
\hline \multicolumn{7}{|l|}{ Severe $(8-10)$} \\
\hline Length, days & $2.5(0.5)$ & $2.2(0.3)$ & & & & 0.50 \\
\hline
\end{tabular}

\section{Discussion}

There are now numerous data showing that bladder urothelium is a complex active sensorial layer able to release several chemical mediators influencing bladder function. ${ }^{20}$ In this context, GAGs would play an important role shaping a protective impermeable layer over whole urothelium, thus inhibiting direct contact between transitional epithelium and urine solutes or microorganisms. ${ }^{21}$

In particular, E. Coli is the most frequent uropathogen associated with $75 \%$ to $90 \%$ and about $60 \%$ of the cases of uncomplicated ${ }^{22,23}$ and complicated UTIs, ${ }^{24}$ respectively. Current evidence suggests that $\mathrm{E}$. coli-infected urine contains soluble factors that can damage the GAGs layer. ${ }^{25}$ Moreover, in individuals with an impaired bladder mucosa, E. Coli colonization and growth is even easier, because the repairing process has been not completed. ${ }^{26}$ Of many others, these studies may lead to consider GAGs layer as a likely target for medications against bacterial UTIs, and for other chronic bladder diseases as well.

Current findings provide clinical evidence that GAGs replacement therapy can be used to prevent urinary infection in women complaining of recurrent UTIs. ${ }^{14-17}$ Indeed we observed both a significant reduction of rate of recurrent UTIs per year and an increased time-to-recurrence, along with an improvement of urinary symptoms. These improvements persisted for 12 months after the end of treatment, with a subsequent worsening. Furthermore, the regression analysis confirmed previously recognized risk factors for UTI episodes thus indicating women over 50 and those with $>4$ UTI episodes per year at higher risk, and thus likely to receive the highest benefit from a prophylactic intravesical GAGs therapy.

Few prospective randomized controlled trials showed that intravesical GAGs therapy could be considered a non-antibiotic alternative therapy for women with recurrent UTIs. ${ }^{14-17}$ Considering the largest cohort of patients ever reported, our findings confirm the results of 3 previously published reports supporting the role of GAGs layer repair as a valid treatment option for bacterial recurrent UTIs.

The "restoring mechanism" was hypothesized as the main prophylactic mechanism of GAGs; ;,14-16 however, a further "antagonist mechanism" of GAGs therapy can also explain the reduction of UTI episodes. Kamhi and colleagues ${ }^{27}$ recently highlighted that GAGs therapy represents the first

\begin{tabular}{|c|c|c|c|}
\hline Variables & OR & $p$ value & IC (95\%) \\
\hline $\begin{array}{l}\text { Age: } 10 \text { years more (from } 20 \\
\text { to } 80 \text { ) }\end{array}$ & 1.14 & 0.327 & $0.87-1.44$ \\
\hline Age $\geq 50$ years & 3.41 & 0.003 & $1.51-7.71$ \\
\hline UTI per year ( $\geq 4$ episodes) & 3.31 & 0.003 & $1.51-7.22$ \\
\hline Elevated BMI status (>25) & 1.21 & 0.598 & $0.5-2.5$ \\
\hline Constipation & 1.1 & 0.792 & $0.51-2.40$ \\
\hline
\end{tabular}

UTI: urinary tract infection; HA: hyaluronic acid; CS: chondroitin sulphate; BMl: body mass index. 
interface for host-pathogen interaction, as well as a coreceptor for bacteria. Rouschop and colleagues ${ }^{28}$ showed that $\mathrm{E}$. Coli invades host cells binding cellular surface HA, and then by CD44-HA interaction. CD44 is a family of type I transmembrane glycoproteins with a wide tissue distribution implicated in many physiological and pathological processes, thus including cytokines/chemokines secretion in the urothelium itself. Therefore, it appears reasonable to speculate that intravesical GAGs instillations may act as a competitive "bacterial glue."

The key GAGs role in protecting from bacterial rUTIs also lies in the potential gradual growing of antimicrobial resistance and the potential side effects of long antibiotic prophylaxis. Until the late 1990s, E. Coli was relatively susceptible to first-line antibiotics, including cephalosporins, fluoroquinolones and trimethoprim-sulfamethoxazole. More recently, several surveillance studies have shown an increasing resistance rate (between $20 \%$ and $45 \%$ ) toward those medications. ${ }^{29}$ Furthermore, prolonged antibiotic use as a prophylactic approach toward recurrent UTIs is eventually burdened by a greater risk of side effects, such as vaginal and oral candidiasis and gastrointestinal symptoms. ${ }^{5}$ Therefore, novel non-antibiotic prophylaxis therapies would be desirable. Cranberry and probiotics are the further non-antibiotic measures available for recurrent UTIs prophylaxis with low efficacy evidence. ${ }^{1}$

Our study has its limitations, including its retrospective design and lack of a control group. However, participating centres adopted a similar treatment protocol, including a comparable follow-up schedule. We included a security profile for GAGs therapy in a larger sample than in previous studies. ${ }^{14-17}$ Moreover, we included a representative image of recurrent UTIs in clinical practice. Furthermore, we described the real-life disease impact on patient QoL.

During past years the management of recurrent UTIs in the field of urothelium GAGs has continuously evolved. ${ }^{30}$ It would seem reasonable to gather more knowledge of GAGs' role over infections.

\section{Conclusion}

This study suggests that intravesical HA-CS administration is an effective and well-tolerated non-antibiotic treatment option in women with recurrent UTI.

Competing interests: Dr. Cicione, Dr. Cantiello, Dr. Ucciero, Dr. Salonia, Dr. Torella, Dr. De Sio, Dr. Autorino, Dr. Carbone, Dr. Romancik, Dr. Tomaskin and Dr. Damiano all declare no competing financial or personal interests.

This paper has been peer-reviewed.

\section{References}

1. Grabe M, Bierklund-Johansen TE, Botto H, et al. European Urological Association. Guidelines on Urological Infections; 2013. httrp://www.uroweb.org/gls/pdf/19\%20Urological\%20infections_LR.pdf. Accessed October 14, 2014.

2. Wagenlehner $F M$, Weidner $W$, Naber KG. An update on uncomplicated urinary tract infections in women. Curr Opin Urol 2009;19:368-74. http://dx.doi.org/10.1097/MOU.0b013e32832ae18c

3. Foxman B, Gillespie B, Koopman J, et al. Risk factors for second urinary tract infection among college women. Am J Epidemiol 2000;151:1194-205. http://dx.doi.org/10.1093/oxfordjournals.aje. a010170

4. Czaja CA, Stamm WE, Stapleton AE, et al. Prospective cohort study of microbial and inflammatory events immediately preceding Escherichia coli recurrent urinary tract infection in women. J Infect Dis 2009;:200:528-36. http://dx.doi.org/10.1086/600385

5. Albert $X$, Huertas I, Pereiro II, et al. Antibiotics for preventing recurrent urinary tract infection in non-pregnant women. Cochrane Database Syst Rev 2004;CD001209.

6. Arnold LD, Bachmann GA, Rosen R, et al. Assessment of vulvodynia symptoms in a sample of US women: A prevalence survey with a nested case control study. Am I Obstet Gynecol 2007;196:128-6. http:// dx.doi.org/10.1016/i.ajog.2006.07.047

7. Arya LA, Northington GM, Asfaw $T$, et al. Evidence of bladder oversensitivity in the absence of an infection in premenopausal women with a history of recurrent urinary tract infections. BJU Int 2012;110:247-51. htrp://dx.doi.org/10.1111/i.1464-410X.2011.10766.x

8. Esko JD, Kimata K, Lindahl U. Proteoglycans and Sulfated Glycosaminoglycans. In: Varki A, Cummings RD, Esko JD, et al., editors. Essentials of Glycobiology. 2nd edition. Cold Spring Harbor (NY): Cold Spring Harbor Laboratory Press; 2009. Chapter 16. Available from: http://www.ncbi.nlm.nih.gov/books/ NBK1900/. Accessed October 14, 2014.

9. Damiano R, Cicione A. The role of sodium hyaluronate and sodium chondroitin sulphate in the management of bladder disease. Ther Adv Urol 201 1;3:223-32. http://dx.doi.org/10.1177/1756287211418723

10. Parsons $\mathrm{CL}$, Greenspan C, Mulholland SG. The primary antibacterial defense mechanism of the bladder. Invest Urol 1975;13:72-8.

11. Parsons CL. New concepts in interstitial cystitis. Int Urogynecol J Pelvic Floor Dysfunct 1997;8:1-2. http:// dx.doi.org/10.1007/BF01920285

12. Lazzeri M, Montorsi F. The therapeutic challenge of "chronic cystitis:" search well, work together, and gain results. Eur Urol 2011;60:78-80. http://dx.doi.org/10.1016/i.eururo.2011.03.039

13. Costantini E, Lazzeri M, Pistolesi D, et al. Morphological changes of bladder mucosa in patients who underwent instillation with combined sodium hyaluronic acid-chondroitin sulphate (laluril). Urol Int 2013;91:81-8. http://dx.doi.org/10.1159/000345047

14. De Vita D, Giordano S. Effectiveness of intravesical hyaluronic acid/chondroitin sulfate in recurrent bacterial cystitis: A randomized study. Int Urogynecol J 2012;23:1707-13. http://dx.doi.org/10.1007/ s00192-012-1794-z

15. Lipovac $M$, Kurz C, Reithmayr F, et al. Prevention of recurrent bacterial urinary tract infections by intravesical instillation of hyaluronic acid. Int J Gynaecol Obstet 2007;96:192-5. http://dx.doi.org/10.1016/i. ïg0.2006.11.025

16. Constantinides C, Manousakas T, Nikolopoulos P, et al. Prevention of recurrent bacterial cystitis by intravesical administration of hyaluronic acid: A pilot study. BJU Int 2004;93:1262-6. http://dx.doi. org/10.1111/j.1464-410X.2004.04850.x

17. Damiano $R$, Quarto $G$, Bava I, et al. Prevention of recurrent urinary tract infections by intravesical administration of hyaluronic acid and chondroitin sulphate: A placebo-controlled randomised trial. Eur Urol 2011:59:645-51. http://dx.doi.org/10.1016/i.eururo.2010.12.039

18. Parsons CL, Dell J, Stanford EJ, et al. Increased prevalence of interstitial cystitis: Previously unrecognized urologic and gynecologic cases identified using a new symptom questionnaire and intravesical potassium sensitivity. Urology 2002;60:573-8. http://dx.doi.org/10.1016/S0090-4295(02)01829-0

19. The National Institute of Diabetes and Digestive and Kidney Diseases (NIDDK). http://www2.niddk.nih. gov/. Accessed October 14, 2014

20. Birder LA, de Groat WC. Mechanisms of disease: Involvement of the urothelium in bladder dysfunction. Nat Clin Pract Urol 2007;4:46-54. http://dx.doi.org/10.1038/ncpuro0672

21. Parsons CL. The therapeutic role of sulfated polysaccharides in the urinary bladder. Urol Clin North Am 1994:21:93-100.

22. Foxman B, Brown P. Epidemiology of urinary tract infections: Transmission and risk factors, incidence, and costs. Infect Dis Clin North Am 2003;17:227-41. http://dx.doi.org/10.1016/S0891$5520(03) 00005-9$

23. Nicolle LE. Uncomplicated urinary tract infection in adults including uncomplicated pyelonephritis. Urol Clin North Am 2008;35:1-12, v. http://dx.doi.org/10.1016/j.ucl.2007.09.004 
Intravesical GAGs treatment in patients with recurrent UTIs

24. Nicolle LE. Complicated urinary tract infection in adults. Can I Infect Dis Med Microbiol 2005;16:349-60.

25. Tay H, Parsons CL, Stein PC. Electrophysiologic monitoring of the effects of soluble virulence factors produced by Escherichia coli infection in urine. Urology 1996;48:389-92. http://dx.doi.org/10.1016/ S0090-4295(96)00209-9

26. Aslan G, Esen N, Esen AA, et al. Recovery period of the bladder after exposure to soluble virulence factor produced by Escherichia coli. Eur Urol 2001;40:446-50. http://dx.doi.org/10.1159/000049814

27. Kamhi E, Joo EJ, Dordick JS, et al. Glycosaminoglycans in infectious disease. Biol Rev Camb Philos Soc 2013;88:928-43. http://dx.doi.org/10.1111/brv.12034

28. Rouschop KM, Sylva M, Teske GJ, et al. Urothelial CD44 facilitates Escherichia coli infection of the murine urinary tract. J Immunol 2006;177:7225-32. http://dx.doi.org/10.4049/iimmunol.177.10.7225
29. Foxman B. The epidemiology of urinary tract infection. Nat Rev Urol 2010;7:653-60. http://dx.doi. org/10.1038/nrurol.2010.190

30. Scott RA, Panitch A. Glycosaminoglycans in biomedicine. Wiley Interdiscip Rev Nanomed Nanobiotechnol 2013;5:388-98. http://dx.doi.org/10.1002/wnan.1223

Correspondence: Dr. Antonio Cicione, Magna Graecia University, Catanzaro, Italy; acicione@libero.it; cicione@unicz.it 